\title{
Attenuation of Frontostriatal Connectivity During Reward Processing Predicts Response to Psychotherapy in Major Depressive Disorder
}

\author{
Erin Walsh', Hannah Carl'2, Tory Eisenlohr-Moul ${ }^{3}$, Jared Minkel ${ }^{3,4}$, Andrew Crowther ${ }^{5}$, Tyler Moore ${ }^{3}$, \\ Devin Gibbs ${ }^{3}$, Chris Petty ${ }^{6}$, Josh Bizzell ${ }^{6,7}$, Moria J Smoski ${ }^{4}$ and Gabriel S Dichter, ${ }^{*, 3,4,5,6,7}$ \\ 'Department of Physical Medicine and Rehabilitation, Program on Integrative Medicine, University of North Carolina at Chapel Hill School of \\ Medicine, Chapel Hill, NC, USA; ${ }^{2}$ Department of Psychology and Neuroscience, Duke University, Durham, NC, USA; ${ }^{3}$ Department of Psychiatry, \\ University of North Carolina at Chapel Hill School of Medicine, Chapel Hill, NC, USA; ${ }^{4}$ Department of Psychiatry and Behavioral Sciences, Duke \\ University Medical Center, Durham, NC, USA; ${ }^{5}$ UNC Neurobiology Curriculum, University of North Carolina at Chapel Hill School of Medicine, \\ Chapel Hill, NC, USA; ${ }^{6}$ Duke-UNC Brain Imaging and Analysis Center, Duke University Medical Center, Durham, NC, USA; ${ }^{7}$ Carolina Institute for \\ Developmental Disabilities, University of North Carolina at Chapel Hill School of Medicine, Chapel Hill, NC, USA
}

\begin{abstract}
There are few reliable predictors of response to antidepressant treatments. In the present investigation, we examined pretreatment functional brain connectivity during reward processing as a potential predictor of response to Behavioral Activation Treatment for Depression (BATD), a validated psychotherapy that promotes engagement with rewarding stimuli and reduces avoidance behaviors. Thirty-three outpatients with major depressive disorder (MDD) and 20 matched controls completed two runs of the monetary incentive delay task during functional magnetic resonance imaging after which participants with MDD received up to I5 sessions of BATD. Seedbased generalized psychophysiological interaction analyses focused on task-based connectivity across task runs, as well as the attenuation of connectivity from the first to the second run of the task. The average change in Beck Depression Inventory-II scores due to treatment was 10.54 points, a clinically meaningful response. Groups differed in seed-based functional connectivity among multiple frontostriatal regions. Hierarchical linear modeling revealed that improved treatment response to BATD was predicted by greater connectivity between the left putamen and paracingulate gyrus during reward anticipation. In addition, MDD participants with greater attenuation of connectivity between several frontostriatal seeds, and midline subcallosal cortex and left paracingulate gyrus demonstrated improved response to BATD. These findings indicate that pretreatment frontostriatal functional connectivity during reward processing is predictive of response to a psychotherapy modality that promotes improving approach-related behaviors in MDD. Furthermore, connectivity attenuation among reward-processing regions may be a particularly powerful endophenotypic predictor of response to BATD in MDD.

Neuropsychopharmacology (2017) 42, 83I-843; doi:I0.1038/npp.2016.179; published online 19 October 2016
\end{abstract}

\section{INTRODUCTION}

Unipolar major depressive disorder (MDD) is the leading cause of global burden among all psychiatric and neurological disorders (Collins et al, 2011) and is one of the leading contributors to global disease burden (Ferrari et al, 2013). Despite the societal costs of MDD, the overall efficacy of antidepressant treatments has not improved substantially in recent years (Undurraga and Baldessarini, 2012): first-line FDA-approved pharmacotherapies demonstrate average response rates of $54 v s 37 \%$ for placebo (Levkovitz et al, 2011), with similar response rates to psychotherapy (Butler

\footnotetext{
*Correspondence: Dr GS Dichter, Department of Psychiatry, University of North Carolina at Chapel Hill School of Medicine, CB 7155, Chapel Hill, NC 27599-7I55, USA, Tel: + I 919445 0132, Fax: + I 919966 4520, E-mail: dichter@med.unc.edu

Received I5 March 2016; revised 22 August 2016; accepted 29 August 2016; accepted article preview online 2 September 2016
}

et al, 2006). Given the suboptimal response to first-line MDD treatments, there has been a growing emphasis on developing methods to match specific subgroups of patients with optimal treatment options (eg, Dunlop et al, 2012; McGrath et al, 2013).

To date, one of the most consistent neural aberrations in MDD is differences in functional neural responses to rewards that may contribute to the core symptom of anhedonia in MDD (Phillips et al, 2015). MDD is characterized by decreased responsiveness of frontostriatal brain regions to rewarding stimuli, including decreased anticipation of forthcoming rewards, reduced pleasure derived from reward presentation, and impaired reward-based learning (eg, Admon and Pizzagalli, 2015 and Der-Avakian and Markou, 2012). Recent efforts to understand the pathophysiology of MDD and treatment response in MDD have shifted from focus on brain activation patterns to identifying distributed synchronous brain networks implicated in core symptom dimensions of MDD (Kaiser et al, 2015; Northoff, 2016), and 
a significant body of this research has addressed resting state connectivity predictors of antidepressant treatment response (see Dichter et al (2014) for a review). However, consistent with the NIMH Research Domain Criteria initiative, focusing on responses to specific classes of stimuli may be particularly useful for identifying the clinically and pathophysiologically meaningful MDD endophenotypes as a means to parse the heterogeneity of MDD. Examining responses to rewards is one such approach, and in the present study, we investigated whether pretreatment functional brain connectivity during reward processing predicted response to Behavioral Activation Treatment for Depression (BATD) psychotherapy using functional magnetic resonance imaging (fMRI). BATD was originally developed to ameliorate symptoms of MDD by promoting sustained interactions with potential reinforcers and inhibiting avoidance behaviors (Hopko et al, 2003; Jacobson et al, 2001), and in this regard, BATD is particularly well suited to ameliorate MDD symptoms overall as well as anhedonia symptoms specifically. In addition, given its specific focus on increased reward-oriented behaviors, BATD provides an ideal mechanistic probe to investigate neural mechanisms related to reward processing and anhedonia in MDD. We recently reported the task-related brain activation results from this sample (Carl et al, 2016), and the focus of the present investigation was to investigate task-related connectivity patterns as potential predictors of response to treatment.

Given prior findings showing linkages between anhedonia and decreased connectivity of the striatum with subcortical and prefrontal brain regions in MDD (eg, Redlich et al, 2015), our primary hypotheses were that pretreatment frontostriatal connectivity would predict the magnitude of clinical response to BATD. However, not all neuroimaging studies have consistently reported decreased frontostriatal response to rewards in MDD (Knutson et al, 2008; Mitterschiffthaler et al, 2003; Schaefer et al, 2006). One recent conceptualization of hedonic functioning in MDD that potentially addresses such inconsistencies is that MDD may be characterized by greater attenuation of response to rewards over time (Pizzagalli et al, 2008). In support of this framework, an investigation of hedonic response duration reported that participants with MDD demonstrated decreased capacity to sustain nucleus accumbens (NAcc) activation during conscious upregulation of positive emotions across the scan session (Heller et al, 2009). Furthermore, the degree of decreased NAcc activity predicted the magnitude of self-reported positive affect in the MDD sample. In a follow-up study, Heller and colleagues reported that the magnitude of change in positive affect following 2 months of treatment with fluoxetine or venlafaxine was associated with sustained activation (ie, less attenuation) of the NAcc and connectivity of the NAcc with the left middle frontal gyrus during upregulation of positive emotions. Thus, a secondary aim of the present study was to evaluate whether attenuation of functional connectivity during reward processing predicted response to BATD. One hypothesis is that relatively preserved reward network function would be predictive of better response to BATD. That is, greater capacity to maintain frontostriatal connectivity (ie, less attenuation) to rewards over time may capitalize on patients' strengths, allowing them to more easily engage with treatment targets, thereby reducing symptoms. An alternative, competing hypothesis is that greater deficits in reward processing (ie, greater connectivity attenuation to rewards over time) would be predictive of more favorable treatment response. Because BATD is a treatment that targets reward-processing deficits, patients with greater impairment may benefit the most, reflecting a remediation of deficits following treatment. By presenting two consecutive runs of the reward task, we were able to evaluate changes in neural connectivity from the first and second task run as potential predictors of BATD response, and thereby test these competing hypotheses. We were specifically interested in whether changes in frontostriatal connectivity between task runs would predict declines in symptoms of depression, and in particular anhedonia, given the role of frontostriatal connectivity in the pathophysiology of anhedonia (Forbes and Dahl, 2012; Heller et al, 2009, 2013).

\section{MATERIALS AND METHODS}

\section{Participants}

As described in Carl et al (2016), this protocol was approved by the Institutional Review Boards at Duke University Medical Center and the University of North Carolina at Chapel Hill, and all participants provided written informed consent. Participants with MDD were recruited via the Cognitive Behavioral Research and Treatment Program at Duke University Medical Center and control participants were recruited via listservs at Duke University and UNCChapel Hill. The MDD and control groups participated in the MRI scan session, after which the MDD group began psychotherapy by study clinicians who received weekly supervision and team didactics in BATD. Up to 15 sessions of BATD were offered; MDD participants received an average of 11.67 ( $\mathrm{SD}=4.40$; range: $2-15)$ weekly sessions. Early responders were given the option to end therapy after a minimum of eight sessions and non-responders received the maximum number of sessions before being referred to the community for additional treatment. Notably, controlling for the number of weeks in treatment did not alter any results presented in this manuscript. A description of BATD is provided in Supplementary Materials I.

Participants in the MDD group met DSM-IV criteria for a current episode of MDD using the Structured Clinical Interview for DSM-IV-TR Axis I Disorders (SCID; First et al, 2002) and scored $\geqslant 15$ on the Hamilton Rating Scale for Depression (HAMD; Hamilton, 1960; Miller et al, 1985), given by trained clinicians under the supervision of a licensed psychologist. Control group participants scored $\leqslant 6$ on the HAMD and did not meet criteria for a current or lifetime episode of a mood disorder. Exclusion criteria included: (1) history of psychosis or mania; (2) active suicidal ideation, (3) evidence of organicity, (4) magnetic resonance imaging contraindication, (5) history of neurological injury or disease, (6) current pregnancy, and, in the MDD group, (7) current mood, anxiety, psychotic, or substance abuse disorder beyond unipolar MDD or dysthymia.

Thirty-eight outpatients with MDD (11 male; mean (SD) age $=33(7.1)$ ) and 20 matched controls (6 male; mean (SD) age $=31(8.8))$ enrolled. Two MDD participants did not return for psychotherapy after the first imaging session and 
were excluded from analyses. Three MDD participants taking psychoactive medications were also excluded from analyses. Thus, the final sample included 33 outpatients with MDD and 20 nondepressed control participants. Of the final sample, 13 MDD participants discontinued treatment early (6 responders (18\%); 7 non-responders $(21 \%))$. Groups did not differ in age, estimated IQ (measured by the North American Adult Reading Test (Blair and Spreen, 1989; NAART), or gender distribution, $p$ 's $>0.32$ (see Supplementary Materials II for participant characteristics). Participants completed a number of different imaging protocols, and results of resting state scans from these control participants and $24 \mathrm{MDD}$ participants have been reported previously (Crowther et al, 2015).

\section{Monetary Incentive Delay fMRI Task}

As described in Carl et al, 2016, during scanning, participants completed two runs of the Monetary Incentive Delay (MID) task (Knutson et al, 2001). Participants practiced the fMRI task outside the scanner before the scan session. During this practice session, participant-specific average reaction times were used to adjust starting reaction times during the scans. Each trial consisted of: (1) a $2000 \mathrm{~ms}$ cue that indicated whether a fast enough response ( $a$ 'hit') to the forthcoming target bullseye could result in a 'reward' (a triangle) or 'no reward' (a circle); (2) a delay period during which a crosshair was presented for 2000-2500 ms ('anticipation phase'); (3) a target bullseye that required a speeded button press presented for up to $500 \mathrm{~ms}$; (4) $3000 \mathrm{~ms}$ of feedback that indicated whether that trial resulted in a 'reward' or not ('outcome phase'); and (5) a variable length ITI crosshair presented such that the total duration of each trial was $12 \mathrm{~s}$. Potential reward and non-potential reward trials were aperiodic and pseudorandomly ordered (Knutson et al, 2000). Participants could win $\$ 2$ per trial and were given feedback about the amount of money won on each trial (eg, ' $+\$ 2$ '). Coincident with feedback, a cumulative total of dollars won within the run was presented. Participants were instructed to respond to target bullseye as quickly as possible, and outcomes were contingent on reaction times. The task was adaptive such that participants were successful on approximately two-third of trials. Each 8-min run contained 40 trials: 20 were potential reward trials and 20 were nonreward trials. Functional connectivity during the anticipation (delay period) and outcome (feedback period) phases were evaluated separately.

\section{Imaging Methods and fMRI Preprocessing}

Functional images were acquired at the Duke-UNC Brain Imaging and Analysis Center (BIAC) on a General Electric (Waukesha, WI) MR750 $3.0 \mathrm{~T}$ scanner equipped with $50 \mathrm{mT} / \mathrm{m}$ gradients $(200 \mathrm{~T} / \mathrm{m} / \mathrm{s}$ slew rate) and an eightchannel head coil for parallel imaging. High-resolution T1-weighted anatomical images were acquired with 162 axial slices using a FSPGR pulse sequence $(\mathrm{TR}=7.584 \mathrm{~ms}$; $\mathrm{TE}=2.936 \mathrm{~ms} ; \mathrm{FOV}=256 \mathrm{~mm}$; image matrix $=256 \times 256$; voxel size $=1 \times 1 \times 1 \mathrm{~mm}$; flip angle $=12^{\circ}$ ) used for normalization and coregistration. This structural image was aligned in a near axial plane defined by the anterior and posterior commissures. Whole-brain functional images were acquired using a spiral-in SENSE sequence $(\mathrm{TR}=1500 \mathrm{~ms}$; $\mathrm{TE}=30 \mathrm{~ms} ; \mathrm{FOV}=240 \mathrm{~mm}$; image matrix, $64 \times 64$; flip angle $=60^{\circ}$; voxel size, $3.75 \times 3.75 \times 4.0 \mathrm{~mm}$; 34 axial slices) to reduce susceptibility artifacts and recover signal in orbital frontal regions (Pruessmann et al, 2001; Truong and Song, 2008). A semi-automated high-order shimming ensured global field homogeneity.

The first four volumes of each functional run were discarded. Data were preprocessed using FSL version 5.0.1 (Oxford Centre for Functional Magnetic Resonance Imaging of the Brain (FMRIB), Oxford University, UK). Preprocessing was applied in the following steps: (i) brain extraction for non-brain removal (Smith et al, 2004), (ii) motion correction using MCFLIRT (Smith, 2002), (iii) spatial smoothing using a Gaussian kernel of FWHM $5 \mathrm{~mm}$, (iv) mean-based intensity normalization of all volumes by the same factor, and (v) high-pass filtering (Jenkinson et al, 2002). Functional images of each participant were coregistered to structural images in native space, and structural images were normalized into a standard stereotaxic space (Montreal Neurological Institute) for intersubject comparison. The same transformation matrices used for structural-to-standard transformations were then used for functional-to-standard space transformations of co-registered functional images. All registrations used an intermodal registration tool (Jenkinson et al, 2002; Smith et al, 2004). Voxel-wise temporal autocorrelation was estimated and corrected using FMRIB's Improved Linear Model (Jenkinson and Smith, 2001).

\section{Treatment Outcome Measures}

Treatment outcomes were evaluated by the Beck Depression Inventory-II (BDI; Beck et al, 1996), collected at the scan session, every 2 weeks during treatment, and at the last psychotherapy session. BDI scores of $0-13$ indicate minimal MDD symptoms, 14-19 indicates mild MDD symptoms, 20-28 indicates moderate MDD symptoms, and 29-63 indicates severe MDD symptoms (Beck et al, 1996). The BDI provides an overall measure of MDD severity and includes items that tap multiple MDD symptom dimensions. We examined BDI total scores, as well as BDI anhedonia subscale scores derived from items $4,12,15$, and 21 (Joiner et al, 2003). Reliability of the BDI was evaluated in a multilevel framework as described by Cranford et al, 2006. Reliabilities of between-person differences across all time points as measured using the BDI total score $\left(R_{\mathrm{KF}}=0.91\right)$ and anhedonia scale $\left(R_{\mathrm{KF}}=0.95\right)$ were excellent; reliability of change over time was also acceptable for both the total score $\left(R_{\mathrm{C}}=0.86\right)$ and the anhedonia subscale $\left(R_{\mathrm{C}}=0.71\right)$.

\section{fMRI Data Analysis}

Task-based functional connectivity during reward anticipation and outcome phases was analyzed using a generalized psychophysiological interaction (gPPI) approach due to its improved power, sensitivity, and specificity in detecting a context-dependent functional connectivity (Cisler et al, 2014; McLaren et al, 2012). We used a region-of-interest (ROI) approach to target canonical reward-processing regions. ROI seeds included the NAcc, caudate, putamen, frontal medial cortex, orbitofrontal cortex (OFC), and anterior cingulate 
cortex. These ROIs were defined using the Harvard-Oxford subcortical and cortical structural probabilistic atlases. Frontal medial cortex and anterior cingulate cortex were divided into left and right hemispheric regions using a custom MATLAB script. In addition, a striatum ROI was constructed by combining the caudate, putamen, and NAcc masks. These ROIs are shown in Supplementary Materials III.

Voxel-wise models evaluated whole-brain connectivity with these seeds. For each participant, mean fMRI time courses (ie, physiological regressors) were extracted from seed regions for each task run using fslmeants in FSL, then multiplied by each psychological regressor of interest (ie, task condition: reward and no reward) to form the PPI interaction terms. The gPPI model included physiological and psychological regressors, as well as their interaction terms to describe the unique effect of these interactions above and beyond the main effects of seed time courses and task conditions. Our primary contrast of interest evaluated the difference between connectivity during reward $v s$ nonreward trials (reward $>$ non-reward) for both anticipation and outcome phases of the task. This allows for the characterization of connectivity patterns specific to reward trials during each of these task phases.

Group differences were modeled two ways. First, group differences were evaluated with respect to seed-based connectivity across both task runs ('global connectivity'). Second, group differences in changes in connectivity over time were evaluated with respect to the contrast of (run $1>$ run 2; 'connectivity attenuation'). Thus, connectivity attenuation is defined as the reduction in functional connectivity from run 1 to run 2. Both approaches used mixed effects analyses with Bayesian estimation techniques, using FMRIB Local Analysis of Mixed Effects (Smith et al, 2004). Resulting images were thresholded using $Z>2.3$ with a corrected cluster significance threshold of $p<0.05$. This method of cluster correction, implemented via the cluster thresholding option within FSL FEAT, compares each cluster's estimated significance (from Gaussian random field theory) with the cluster probability threshold to eliminate clusters below this threshold. Betweengroup comparisons were restricted to clusters within corticostriatal regions generated by combining all reward-processing ROIs into a single mask. Clusters were then thresholded at $Z>2.58$ or $p<0.005$ (corrected) with a minimum contiguous cluster size of 8. Cluster localizations were based on HarvardOxford cortical and subcortical structural probabilistic atlases, with Brodmann area identification via Talairach Daemon in FSLView v3.1.8.

\section{Analytic Plan for Predicting Treatment Response from Functional Connectivity}

We used hierarchical linear models (HLMs) to examine whether clusters indicating group differences in connectivity predicted treatment response measured via nine BDI assessments over 15 weeks using SAS PROC MIXED 9.4 (SAS Institute, Cary, NC, USA), with treatment week at level 1 and person at level 2. Treatment week was a continuous time variable and was uncentered. We specified an autoregressive (week 2) covariance structure for withinperson errors. Individual coefficients were presented as gamma weights that were analogous to unstandardized beta coefficients in standard regression, representing the estimated change in the dependent variable given a one-unit increase in the predictor. For further details about our HLM approach, please see Supplementary Materials IV.

\section{Motion Correction}

Motion can have a particularly strong impact on connectivity analyses (Power et al, 2012). All runs from all participants had motion that was $<5 \mathrm{~mm}$ along any of the six possible axes, ie, $x, y, z$, pitch, yaw, and roll (with the exception of one dimension for a single run for one control participant). There were no significant effects of group or run for mean or max absolute head motion along any of the six possible axes, all $p$ 's $>.05$. Furthermore, we tested for group differences in absolute root mean squared (RMS) displacement. Results showed there was no significant difference between MDD and Control participants across runs, $p>0.05$. Motion plots and analyses can be viewed in Supplementary Materials V. RMS displacement was also entered as a covariate in group-level analyses for seed-based connectivity models where clusters were predictive of treatment response. Tables including RMS displacement as a covariate are presented in the Supplementary Materials (Tables 1 and 2). Because there were no between-group differences in RMS displacement and because the inclusion of this covariate in group-level analyses did not meaningfully alter findings (including HLM models predicting treatment response), this covariate was not included in the other group-level connectivity models.

Table I Group Differences in Global Functional Connectivity (ie, across runs)

Anticipation phase:

\begin{tabular}{|c|c|c|c|c|c|c|}
\hline \multirow[t]{2}{*}{ Seed } & \multirow[t]{2}{*}{ Region (BA) } & \multicolumn{3}{|c|}{ MNI coordinates } & \multirow[t]{2}{*}{$k$} & \multirow{2}{*}{$\begin{array}{l}\text { Peak } \\
\text { z-score }\end{array}$} \\
\hline & & $x$ & $Y$ & $\mathbf{Z}$ & & \\
\hline \multicolumn{7}{|l|}{$M D D>C O N$} \\
\hline \multirow[t]{5}{*}{ Left caudate } & ACC (BA 9/32) & -4 & 26 & 24 & 290 & 3.83 \\
\hline & ACC (BA 24) & 6 & 14 & 28 & 22 & 3.00 \\
\hline & $\begin{array}{l}\text { R paracingulate gyrus } \\
\text { (BA 24/32) }\end{array}$ & 12 & 12 & 44 & 61 & 3.63 \\
\hline & L ACC & -2 & 2 & 38 & 37 & 3.06 \\
\hline & $\begin{array}{l}\text { Paracingulate gyrus } \\
\text { (BA 6/8) }\end{array}$ & 8 & 26 & 42 & 21 & 3.43 \\
\hline \multicolumn{7}{|l|}{$M D D<C O N$} \\
\hline \multirow[t]{5}{*}{ Left OFC } & $\mathrm{L}$ putamen & -28 & -2 & 12 & 13 & 3.14 \\
\hline & $\mathrm{R}$ caudate & 18 & 14 & 14 & 21 & 3.48 \\
\hline & $\mathrm{R}$ caudate & 8 & 2 & 16 & 103 & 3.71 \\
\hline & $\mathrm{L}$ caudate & -16 & 14 & 16 & 19 & 3.20 \\
\hline & $\mathrm{L}$ caudate & -20 & -2 & 20 & 79 & 3.55 \\
\hline Right OFC & $L$ caudate & -12 & -16 & 24 & 54 & 3.21 \\
\hline
\end{tabular}

Abbreviations: $k$, number of voxels in each cluster; BA, Brodmann Area; $\mathrm{R}$, right, $\mathrm{L}$, left; $\mathrm{ACC}$, anterior cingulate cortex; OFC, orbitofrontal cortex. All clusters are thresholded at $p<0.005$ (corrected), $>8$ contiguous voxels; $\mathrm{MNI}$ coordinates reflect the maximum voxel of intensity for each cluster. 
Table 2 Group Differences in Functional Connectivity Attenuation (ie, run I > run 2)

\begin{tabular}{|c|c|c|c|c|c|c|}
\hline \multirow[t]{2}{*}{ Seed } & \multirow[t]{2}{*}{ Region (BA) } & \multicolumn{3}{|c|}{ MNI coordinates } & \multirow[t]{2}{*}{$k$} & \multirow[t]{2}{*}{ Peak z-score } \\
\hline & & $x$ & $Y$ & $Z$ & & \\
\hline
\end{tabular}

Anticipation phase:

MDD $>$ CON

Left striatum

L OFC/FP/FMC (BA II/47)

R OFC/subcallosal cortex/FMC (BA I0/ I /47)

L OFC (BA 25)

R FMC/FP (BA II)

Right striatum

R OFC/subcallosal cortex/temporal pole (BA 25/47)

L OFC/temporal pole (BA I I/25/47)

R FP

R FMC/FP (BA I I)

Left NAcc

Paracingulate gyrus/FP (BA 9/10)

Left caudate

L OFC/FP (BA I I/47)

$\mathrm{L}$ subcallosal cortex

Right caudate

R OFC/subcallosal cortex (BA 25/47)

L OFC/subcallosal cortex/FP (BA I |/25/47)

R FMC/FP

R FMC

Left putamen

L OFC (BA I I/47)

R OFC/subcallosal cortex/temporal pole (BA 5/47)

Subcallosal cortex (BA II)

R FMC/FP (BA I I)

$L F P(B A||)$

FMC

Right putamen

L OFC/temporal pole (BA I I/25/47)

R OFC/subcallosal cortex/temporal pole (BA 25/47)

R temporal pole/OFC (BA 47)

Left ACC R OFC/subcallosal cortex (BA I I/25/47)

Right ACC R OFC (BA 25)

$L$ OFC/FP (BA I I/47)

$L F P$

R FMC/FP (BA 10/1 I)

Right FMC ACC (BA 24/32)

Paracingulate gyrus (BA 32)

Outcome phase:

MDD > CON

Right striatum

R OFC/subcallosal cortex (BA 25/47)

Left NAcc

$\mathrm{L}$ paracingulate gyrus/FP (BA 10)

Paracingulate gyrus (BA 32)

\section{$M D D<C O N$}

Left striatum

Right striatum

Right NAcc

R putamen

R putamen

$R$ putamen

$R$ inferior frontal gyrus (BA 44/45)

$R$ insula

Left caudate

$R$ putamen

R putamen

$R$ insula

Right caudate
12

$-10$

4

18

$-22$

10

4

$-12$

$-22$

$-10$

10

$-22$

10

10

$-30$

14

$-4$

4

$-12$

0

$-22$

14

32

18

14

$-24$

$-10$

12

10

$-12$

14

$-10$

$-14$

34

24

34

48

34

36

26

36

34
$-20$

292

4.12

$-12$

$-20$

$-24$

335

29

3.71

3.11

3.36

4.03

4.46

3.08

3.21

3.77

3.71

2.80

3.54

4.29

2.86

3.42

4.13

3.96

2.90

3.67

3.25

2.76

4.59

4.47

3.74

4.18

3.98

3.56

3.12

4.15

3.47

3.08 
Table 2 Continued

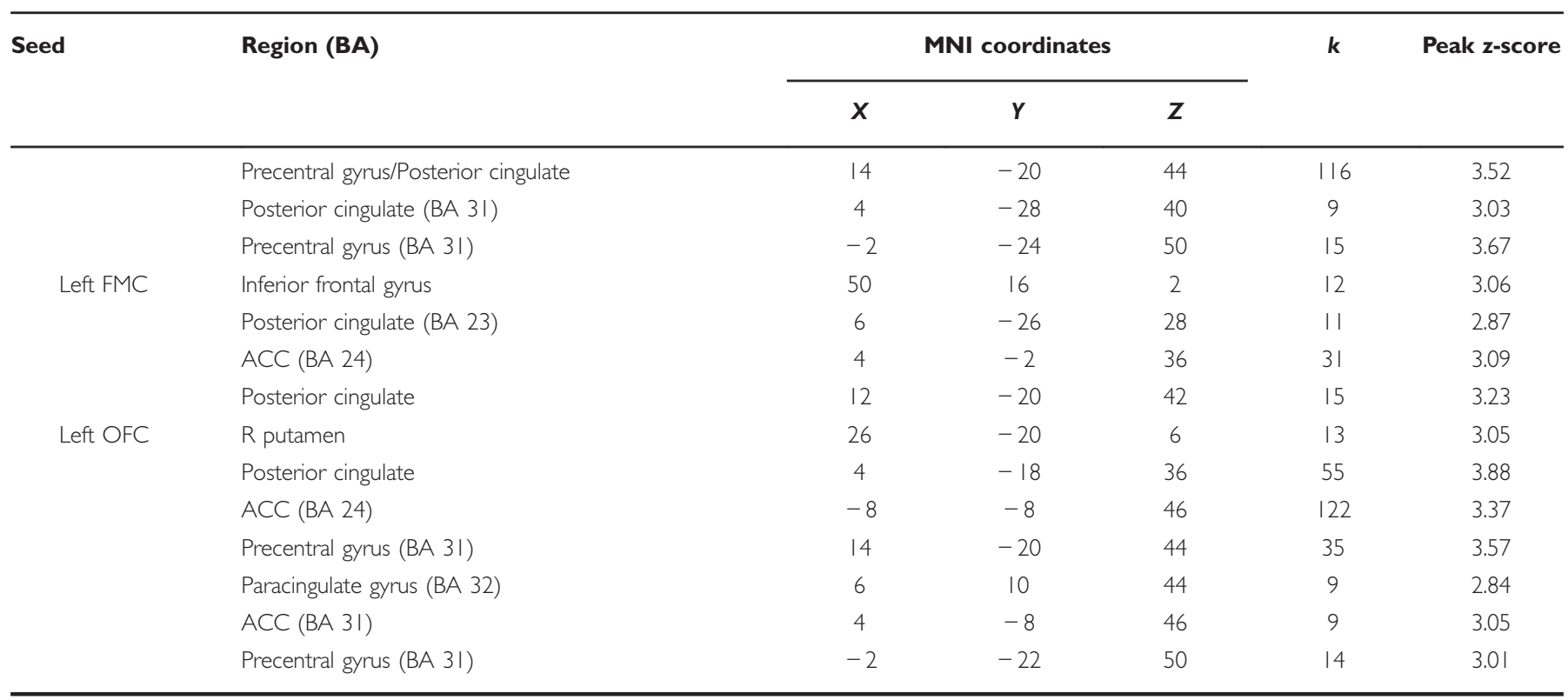

Abbreviations: $k$, number of voxels in each cluster; BA, Brodmann Area; R: right, L, left; ACC, anterior cingulate cortex; FMC, frontal medial cortex; FP, Frontal Pole; OFC, orbitofrontal cortex; NAcc, nucleus accumbens.

Clusters are thresholded at $p<0.005$ (corrected), $>8$ contiguous voxels; MNI coordinates reflect the maximum voxel of intensity for each cluster.

\section{RESULTS}

\section{Effects of Treatment Week on Depression Scores}

Treatment resulted in highly significant reductions in BDI total scores (pretreatment mean $(\mathrm{SD})=25.27(8.52)$, posttreatment mean $(\mathrm{SD})=14.73(9.96), p<0.001)$ and $\mathrm{BDI}$ anhedonia subscale scores (pretreatment mean $(\mathrm{SD})=4.91$ (2.26), post-treatment mean (SD) $=2.87$ (2.00), $p<0.001$ ). This is consistent with results from HLM models that showed a significant main effect of treatment week on BDI total and BDI anhedonia subscale scores, with both showing highly significant linear decreases over time (effect of treatment week on BDI total scores: $\gamma_{\text {TREATMENT }}$ WEEK $=-0.95, \mathrm{SE}=0.12$, $t(202)=-7.69, p=<0.0001$; and on BDI anhedonia subscale scores: $\gamma_{\text {TREATMENT WEEK }}=-0.18, \mathrm{SE}=0.031, t(202)=-5.86$, $p=<0.0001$ ). Supplementary Material VI illustrates biweekly individual patient and group-averaged BDI scores during the course of BATD treatment.

\section{Task-Related Functional Connectivity (gPPI) Results}

Group differences in global connectivity (ie, averaged across runs). Group differences in task-dependent global functional connectivity (ie, across runs) are illustrated in Table 1. During the anticipation phase of the task, the MDD group exhibited increased connectivity, relative to controls, between the left caudate seed and several clusters in the anterior cingulate and paracingulate gyrus, and decreased connectivity between the OFC seeds and clusters in the left and right caudate and left putamen. No group differences were evident in the outcome phase of the task.

Group differences in connectivity attenuation (ie, reductions from run 1 to run 2). Group differences in taskdependent connectivity attenuation (run 1>run 2) during the anticipation and outcome phases of the MID task are shown in Table 2 and select results are illustrated in Figure 1. During the anticipation phase of the task, there were multiple clusters revealing relatively greater attenuation of connectivity in the MDD group in medial anterior portions of the left and right prefrontal cortex, including frontal medial cortex, frontal pole, and OFC with numerous frontostriatal seed regions (eg, striatum and anterior cingulate). In contrast, there were only three clusters showing significantly greater connectivity attenuation in the outcome phase of the MID task in the MDD group: the right striatum seed with a cluster containing portions of right OFC and subcallosal cortex, and the left NAcc seed with clusters in the paracingulate gyrus.

During the outcome phase of the MID task, there were also multiple clusters where the MDD group exhibited relatively less connectivity attenuation. The left and right striatum seeds, the right NAcc seed, and the left and right caudate seeds showed less attenuation of connectivity with overlapping clusters in the right putamen. Furthermore, the right caudate seed, the left frontal medial cortex seed, and the left OFC seed also showed significantly less connectivity attenuation with clusters in anterior and posterior portions of the cingulate cortex and the precentral gyrus.

\section{Task-Related Connectivity as a Predictor of Treatment Response}

For clusters that differentiated groups in terms of global connectivity (Table 1) and connectivity attenuation (Table 2), HLM models were used to evaluate whether individual differences in pretreatment functional connectivity predicted the slope of change in BDI total and anhedonia scores over the course of BATD. 


\section{Global Connectivity as a Predictor of Treatment Response}

Prediction of change in BDI total and anhedonia scores from connectivity between the left caudate seed and right paracingulate gyrus. As illustrated in Figure 2, one cluster that reflected group differences in pretreatment global connectivity was predictive of treatment response: during reward anticipation, greater connectivity between the left caudate seed and right paracingulate gyrus (BA24/32) was associated with greater declines in BDI total and anhedonia subscale scores during treatment (BDI total score: $\gamma_{\text {CONNECTIVITY }}{ }^{*}$ TREATMENT WEEK $=-0.39, \mathrm{SE}=0.19, t(186)=$ $-2.02, \quad p=0.045 ; \quad$ BDI anhedonia subscale score: $\gamma_{\text {CONNECTIVITY }} *$ TREATMENT $\quad$ WEEK $=-0.094, \quad \mathrm{SE}=0.031$, $t(187)=-3.10, p=0.0023)$. Pseudo- $R^{2}$ calculations indicated that this connectivity metric accounted for $\sim 7.6 \%$ of the between-person variance in the within-person effect of treatment week on BDI total scores, and $\sim 25 \%$ of the between-person variance in the within-person effect of treatment week on BDI anhedonia subscale scores. Figure 2 illustrates the trends for BDI total scores, and BDI anhedonia subscale scores reflect the same pattern (not shown). The direction of this effect reflects that patients whose global connectivity patterns were more different from controls (ie, those with greater pretreatment connectivity) fared better after treatment.

\section{Connectivity Attenuation as a Predictor of Treatment Response}

Individual differences in connectivity attenuation between several regions pairs also predicted treatment response. All significant models were for clusters that reflected greater attenuation of connectivity in the MDD group relative to the control group.

Prediction of change in BDI total scores from connectivity attenuation between the right putamen seed and right OFC /temporal pole. As illustrated in Figure 3, during the anticipation phase of the MID task, less connectivity attenuation between the right putamen seed and right OFC /temporal pole (BA47) was associated with greater declines in BDI total scores during treatment $\left(\gamma_{\text {CONNECTIVITY }}\right.$ DIFFERENCE ${ }^{*}$ TREATMENT WEEK $=0.40, \mathrm{SE}=0.14, t(185)=2.80$, $p<0.0001)$; individual differences in connectivity attenuation of these regions accounted for $16 \%$ of the variance in BDI total score change during treatment. The direction of this effect reflects that patients whose connectivity attenuation patterns were more similar to controls (ie, those with less dropoff in connectivity between run 1 and run 2) fared better after treatment.

Prediction of change in BDI total scores from connectivity attenuation between the left NAcc seed and right paracingulate gyrus. As illustrated in Figure 4, during the reward outcome phase of the MID task, greater connectivity attenuation between the left NAcc seed and paracingulate gyrus (BA 32) was associated with greater declines in BDI total scores during treatment $\left(\gamma_{\text {CONNECTIVTTY DIFFERENCE*- }}\right.$ TREATMENT WEEK $=-0.41, \mathrm{SE}=0.16, t(185)=-2.51, p=0.013$ ); individual differences in connectivity attenuation of these regions accounted for $4.7 \%$ of the between-person variance in BDI total score change across treatment (Figure 4). The direction of these effects indicates that patients whose connectivity attenuation patterns were less similar to controls (ie, those with greater dropoff in connectivity between run 1 and run 2) fared better after treatment.

Prediction of change in BDI total scores from connectivity attenuation between the right frontal medial cortex seed and paracingulate gyrus. In addition, greater connectivity attenuation between the right frontal medial cortex seed and paracingulate gyrus (BA 32) during reward anticipation predicted greater reductions in BDI total scores across treatment $\left(\gamma_{\text {CONNECTIVITY DIFFERENCE }}{ }^{*}\right.$ TREATMENT WEEK $=$ $-0.36, \mathrm{SE}=0.14, t(185)=-2.58, p=0.010)$. Individual differences in connectivity attenuation of the right frontal medial cortex seed and paracingulate gyrus (BA32) accounted for $11 \%$ of the between-person variance in BDI total score change. The direction of these effects indicates that patients whose connectivity attenuation patterns were less

\section{a}

Left Striatum Seed Anticipation
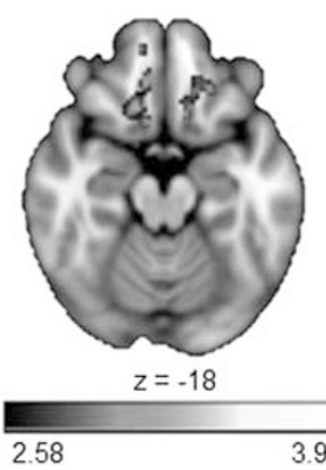

b

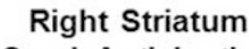
Seed, Anticipation
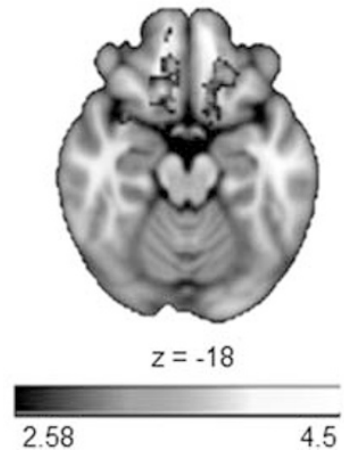

C Right Striatum Seed, Outcome

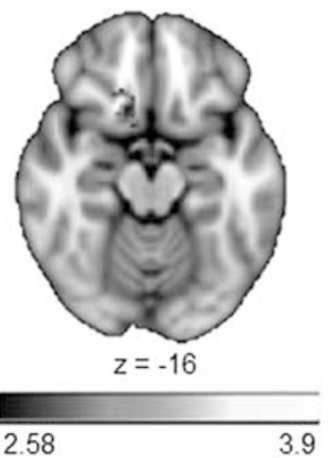

Figure I Group differences in task-dependent connectivity attenuation (run I > run 2) during the anticipation and outcome phases of the MID task. Relative to the control group, the MDD group showed greater connectivity attenuation (run I > run 2) with clusters in the orbitofrontal cortex and subcallosal cortex and (a) the left striatum seed during reward anticipation, (b) the right striatum seed during reward anticipation, and (c) the right striatum seed during reward outcomes. Note that across all three contrast maps, clusters are within the orbitofrontal cortex and subcallosal cortex. A full color version of this figure is available at the Neuropsychopharmacology journal online. 

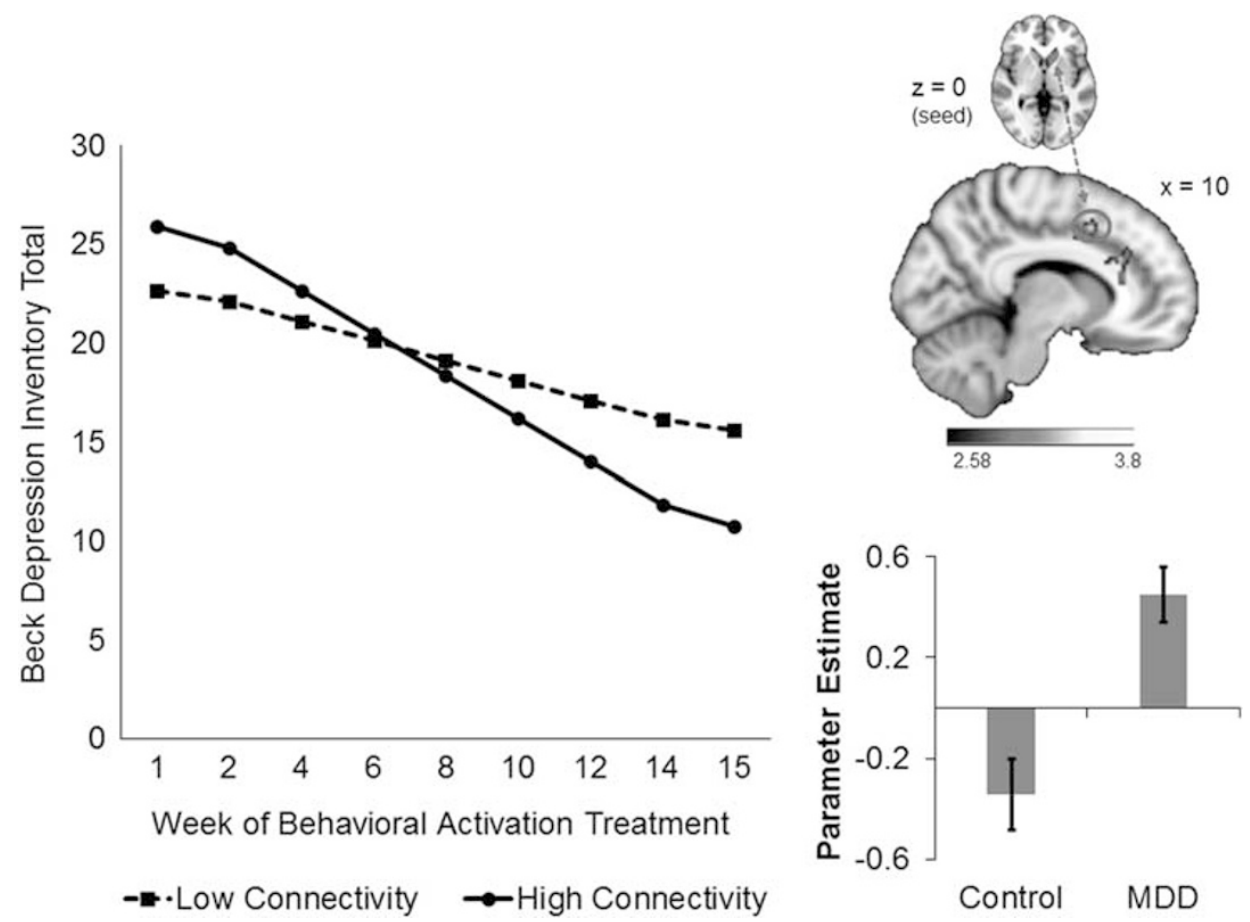

Figure 2 Within the MDD group, greater global connectivity between the left caudate (seed) and right paracingulate gyrus (BA 24/32) during reward anticipation predicted greater improvement in BDI total scores over the course of BATD treatment. The plot is a graphical illustration of the significant interaction between pretreatment connectivity and time predicting change in BDI scores from the HLM models. The lines represent the expectation for change in an individual who is one SD below the mean and one SD above the mean. Note the lines are model-based estimates and do not represent averages but rather ranges of brain connectivity variability. The brain image indicates the seed region above and the connecting cluster beneath. The bar graph illustrates connectivity estimates. A full color version of this figure is available at the Neuropsychopharmacology journal online.

similar to controls (ie, those with greater dropoff in connectivity between run 1 and run 2) fared better after treatment.

Prediction of change in BDI anhedonia scores from connectivity attenuation between the left putamen seed and subcallosal cortex. Greater connectivity attenuation between the left putamen seed and subcallosal cortex (BA11) during reward anticipation (not shown) was also associated with greater declines in BDI anhedonia subscale scores $\left(\gamma_{\text {CONNECTIVITY DIFFERENCE }}{ }^{*}\right.$ TREATMENT WEEK $=-0.08, \mathrm{SE}=$ $0.025, t(186)=-3.20, p=0.0016)$. Individual differences in connectivity attenuation accounted for $33 \%$ of the variance in BDI anhedonia subscale score change across treatment. The direction of these effects indicates that patients whose connectivity attenuation patterns were less similar to controls (ie, those with greater dropoff in connectivity between run 1 and run 2) fared better after treatment.

\section{DISCUSSION}

The purpose of this study was to investigate whether pretreatment functional connectivity during a rewardprocessing task predicted response to BATD in outpatients with MDD. BATD promotes interactions with potentially rewarding activities and decreases withdrawal behaviors (Hopko et al, 2003; Jacobson et al, 2001), thereby making it an ideal mechanistic probe to evaluate relations between connectivity in reward-processing brain regions and response to a treatment modality that increases responses to motivationally relevant information. The clinical effectiveness of BATD in the current study was consistent with prior trials (Dichter et al, 2009; Hopko et al, 2003): average BDI scores declined 10.54 points, a clinically meaningful response (Jacobson and Truax, 1991), a shift from moderate to mild depressive symptoms.

We evaluated both global connectivity (ie, across task runs) and connectivity attenuation (ie, reductions in connectivity from run 1 to run 2). We found increased global connectivity in the MDD group during reward anticipation between the left caudate seed and several clusters in the anterior cingulate and paracingulate gyrus, and decreased global connectivity between the OFC seeds and clusters in the left and right caudate, and left putamen, whereas there were no group differences during the outcome phase of the task. There was also greater connectivity attenuation in the MDD group in medial anterior portions of the left and right prefrontal cortex, including frontal medial cortex, frontal pole, and OFC, during reward anticipation and between striatal seeds and OFC, subcallosal cortex, and paracingulate gyrus during reward outcomes.

Group differences in connectivity between mid-frontal brain regions and the striatum in MDD are consistent with other investigations using resting state approaches (Kaiser et al, 2015) as well as prior reports in participants with remitted MDD (Admon et al, 2015; Morgan et al, 2016), but extend this literature to the context of a reward-processing task (Satterthwaite et al, 2015). These results also extend prior findings on frontostriatal connectivity attenuation in MDD (Heller et al, 2013) and highlight the importance of 

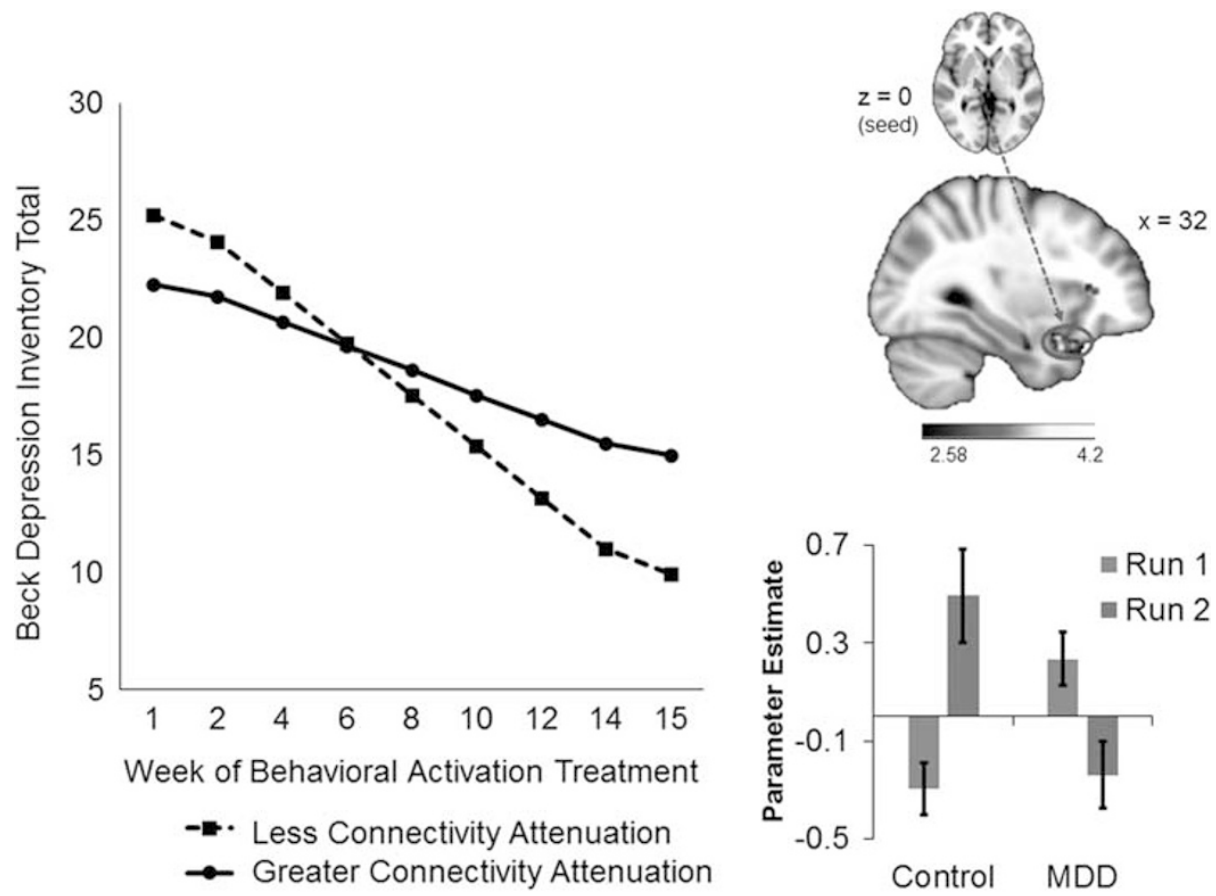

Figure 3 Within the MDD group, less connectivity attenuation between the right putamen (seed) and right orbitofrontal cortex/temporal pole (BA 47) during reward anticipation predicted greater improvement in BDI total scores over the course of BATD treatment. The plot is a graphical illustration of the significant interaction between pretreatment connectivity and time predicting change in BDI scores from the HLM models. The lines represent the expectation for change in an individual who is one SD below the mean and one SD above the mean. Note the lines are model-based estimates and do not represent averages but rather ranges of brain connectivity variability. The brain image indicates the seed region above and the connecting cluster beneath. The bar graph illustrates connectivity estimates. A full color version of this figure is available at the Neuropsychopharmacology journal online.
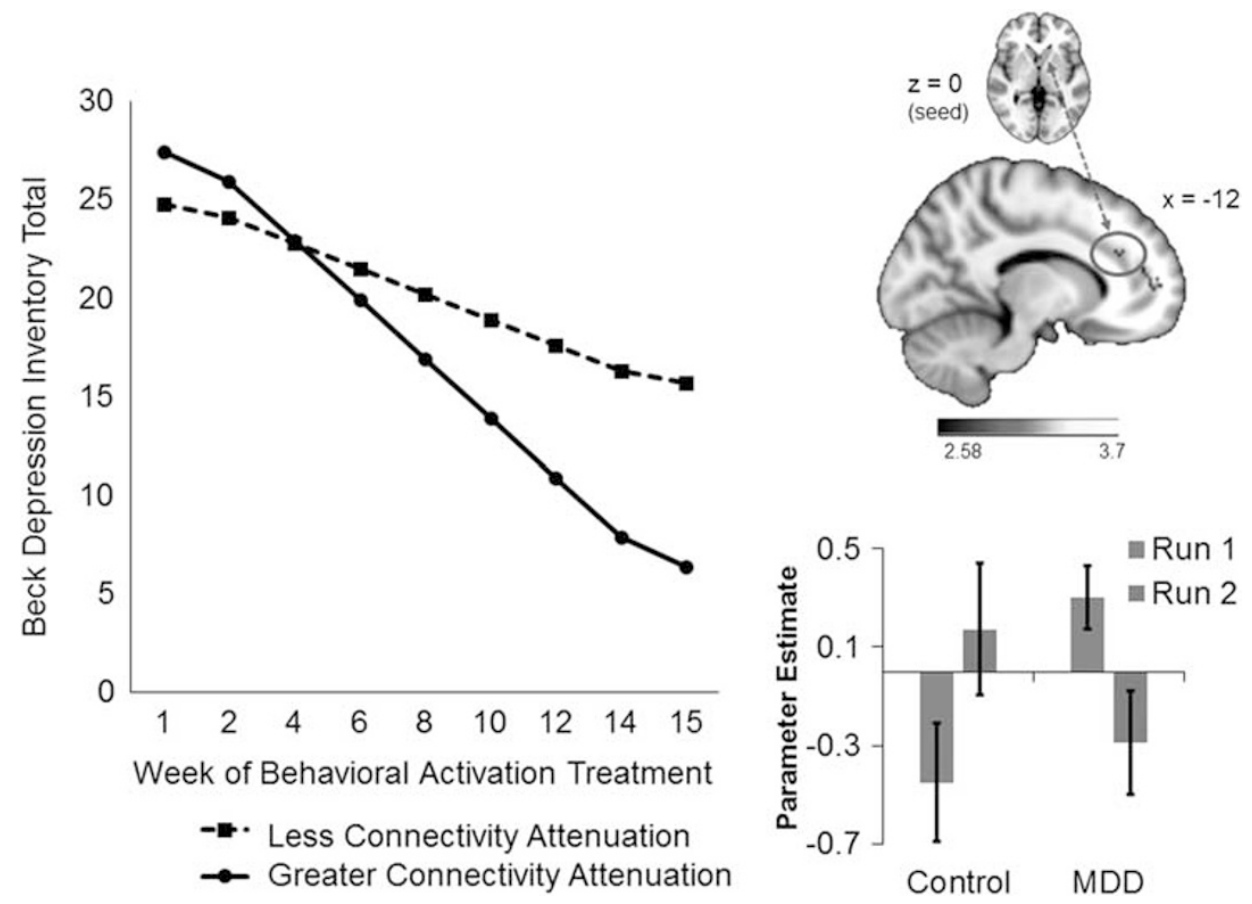

Figure 4 Within the MDD group, greater connectivity attenuation between the left nucleus accumbens (seed) and paracingulate gyrus (BA 32) during reward outcomes predicted greater improvement in BDI total scores over the course of BATD treatment. Findings between the left putamen (seed) and subcallosal cortex (BA II) during reward anticipation and the right frontal medial cortex (seed) and paracingulate gyrus (BA 32) during reward anticipation followed the same pattern. A full color version of this figure is available at the Neuropsychopharmacology journal online. 
examining the temporal dynamics of neural responses for understanding the pathophysiology of MDD (Heller et al, 2009; Pizzagalli et al, 2008). The localization of many connectivity clusters to the OFC, cingulate cortex, and subcallosal cortex further underscores the relevance of altered connectivity in these regions to MDD etiology (Chai et al, 2015; Choi et al, 2015).

We used HLMs to examine whether clusters reflecting group differences in global connectivity and connectivity attenuation in frontostriatal regions predicted response to BATD. The majority of findings showed that patients whose brain connectivity patterns were more divergent than controls responded better to treatment, as evidenced by greater declines in BDI total and/or anhedonia subscale scores. Furthermore, significant clusters were largely localized to regions within/bordering the dorsal anterior cingulate cortex (dACC) and medial prefrontal cortex (mPFC). Both regions have central roles in reward-based decision-making and behavior. The dACC is involved in estimating value of potential rewards and determining the worth of investing cognitive control to a given action (Shenhav et al, 2013; Zhang et al, 2013), whereas the mPFC is linked to subjective value of objects and choices (Glascher et al, 2009; Haber and Knutson, 2010). Thus, greater disturbances in connectivity-and, particularly, rapid attenuation of connectivity-between the striatum and these frontal regions may be related to impairments in reward valuation and goal-directed behavior. Because a key component of BATD is to increase engagement with valued behavior (Dimidjian et al, 2011), it may be the case that this treatment functions especially well for patients with greater deficits in reward-related frontostriatal connectivity. That is, BATD may be effective in remediating deficits associated with anticipatory processing of rewards, effort valuation, and decision-making to engage in goal-directed behavior. This interpretation suggests a framework for understanding a potential pathway to personalized MDD interventions: patients with greater deficits in connectivity, and the capacity to sustain connectivity, in reward-processing regions may be good candidates for treatments that target reward network functioning and related behaviors, whereas other treatments may be more suited to patients with different patterns of neural connectivity. Clearly, larger-scale studies that evaluate patient responses to a number of different treatment modalities will be needed to fully evaluate this framework (McGrath et al, 2014).

Although the majority of our results show that patients with connectivity patterns that were more divergent from controls showed a more improved response to BATD, one connectivity pattern reflected better response in individuals who were more similar to controls. Specifically, less connectivity attenuation between the right putamen seed and right OFC/temporal pole (BA47) during reward anticipation was associated with greater declines in BDI total scores (ie, those with less dropoff in connectivity responded better to treatment). Previous studies have shown MDD to be characterized by altered OFC function during reward selection (Smoski et al, 2009) and receipt of unexpected rewards (Segarra et al, 2016). It may be the case that patients with MDD with some preservation of reward network function (ie, those whose connectivity patterns look more similar to controls) respond better to BATD treatment.
Although the majority of our findings are suggestive of BATD being particularly effective for patients with greater brain reward network disturbances, it is likely that some motivational capacity may be needed to fully benefit from this psychotherapy. This interpretation parallels results from our previous report in this sample (Carl et al, 2016), where we showed that patients with less attenuation of activation in the ACC during reward outcomes were more responsive to BATD. Although MDD and control groups did not differ in activation magnitude in this region, prior research suggests that increased baseline ACC activation is predictive of positive treatment response in $\mathrm{MDD}$ (Fu et al, 2013; Pizzagalli, 2011). Taken together, these findings highlight the relevance of attenuation (or lack thereof) of frontocingulate neural activation and connectivity in predicting response to antidepressant treatment.

The present study evaluated a treatment that targets decreased motivation as a core feature of MDD. Anhedonic symptoms are often the most difficult MDD symptom to treat and may represent a critical vulnerability factor for various psychiatric disorders (Hasler et al, 2004; Pizzagalli et al, 2005). Although the majority of neural connectivity treatment predictors in this study involved BDI total scores, the largest effect sizes were observed for prediction of change in BDI anhedonia subscale scores (ie, BDI anhedonia subscale scores accounted for a larger percent of the between-person variance in treatment response than BDI total scores). This suggests that reward system connectivity may be a particularly robust predictor of changes in anhedonic symptoms.

It is notable that analyses of global connectivity yielded only one finding that was predictive of treatment response, whereas there were four patterns reflective of connectivity attenuation that were predictive of treatment response. This suggests the importance of examining temporal changes in neural connectivity as an MDD endophenotype that is particularly relevant to predicting antidepressant treatment response. In addition, connectivity patterns that differentiated MDD patients from controls, as well as those that were predictive of treatment response, were primarily related to anticipatory processing. Reaction times during rewarded trials-reflecting anticipatory processing/motivation-were also predictive of treatment response (Supplementary Materials VII). These findings suggest that anhedonia in MDD may be more strongly linked to components of appetitive motivation than hedonic processing. Further, neural and behavioral methods for assessing anticipatory processing may be powerful predictors of treatment response in MDD.

Previous studies of sustained neural responses in MDD (Heller et al, 2009, 2013) applied beta series correlation methods wherein individual trials are estimated, whereas the current study used a gPPI approach to average beta estimates across trials for each condition to compare changes in connectivity from the first to the second run of the task. In this regard, the gPPI approach may be interpreted to reflect differential habituation in the MDD group. (We thank an anonymous reviewer for this additional interpretation.) Future work should examine whether habituation to the task or cognitive fatigue arising during multiple administrations may contribute to differential changes in connectivity in MDD during scanning. In addition, our analytic approach 
of evaluating group by run interaction terms emphasizes patterns of connectivity in the MDD group that are opposite to controls. Although this approach was designed to underscore connectivity patterns in the MDD group that were atypical, it is not sensitive to identify patterns where attenuation of connectivity may be similar in both MDD and control groups. Despite these differences in modeling approaches, our findings are consistent with existing evidence (Heller et al, 2009, 2013) and provide additional support for investigating temporal trajectories of brain connectivity patterns.

Limitations. Limitations of the current study to address in future research include incorporating additional task trials (eg, reward loss) or experimental paradigms to more fully assess different aspects of reward processing, such as valence or arousal (eg, Liu et al, 2011). The present study also lacked a comparison treatment condition (eg, a different psychotherapy modality or a psychopharmacologic treatment). In this regard, our findings cannot be attributed specifically to BATD. In addition, post-treatment scans were not acquired, and thus it was not possible to examine the effects of BATD on reward-related connectivity. Likewise, longitudinal clinical follow-up data would be needed to address neural predictors of MDD relapse. Finally, because we recruited based on relatively mild depression scores (HAMD $\geqslant 15$ ), future studies should replicate these findings in samples with greater symptom severity.

\section{CONCLUSIONS}

In summary, the current study found that functional connectivity in reward-processing brain regions in the context of a reward-processing task was predictive of response to BATD, a psychotherapy modality that improves motivation and engagement with patient-specific goals. Evaluation of connectivity attenuation yielded four findings that were predictive of treatment response, underscoring the relevance of the temporal dynamics of frontostriatal connectivity for predicting response to this treatment modality. These findings extend the emerging framework of MDD that emphasizes decreased capacity to sustain neural response to hedonic stimuli in the pathophysiology of the disorder (Carl et al, 2016; Heller et al, 2009, 2013) to the domain of treatment prediction. More generally, combined with our prior examination of resting state predictors of BATD response in a subset of this sample (Crowther et al, 2015) and emerging evidence addressing neural predictors of response to other antidepressant treatment modalities (see Dichter et al, 2014 for a review), these findings contribute to the growing body of literature addressing pretreatment neuroimaging endophenotypes as predictors of antidepressant treatment response (McGrath et al, 2013). The ultimate goal of such studies is to improve response rates to available MDD treatments by developing predictive models of treatment response (Kapur et al, 2012).

\section{FUNDING AND DISCLOSURE}

The project was funded by R21 MH094781 and R21 MH094781 S1 to GSD and MJS, by NIMH K23 MH087754 to MJS, and by NIMH K23 MH081285 to GSD. EW was supported by T32AT003378. TEM was supported by T32MH093315. JB was supported by HD079124. The content is solely the responsibility of the authors and does not necessarily represent the official views of the National Institutes of Health. The authors declare no conflict of interest.

\section{ACKNOWLEDGMENTS}

We thank MRI technologists Susan Music, Natalie Goutkin, and Luke Poole for assistance with data acquisition, and BIAC Director Allen Song for assistance with various aspects of this project. Portions of these results were presented at the 2015 Annual Meeting of the American College of Neuropsychopharmacology in Hollywood, FL.

\section{REFERENCES}

Admon R, Holsen LM, Aizley H, Remington A, WhitfieldGabrieli S, Goldstein JM et al (2015). Striatal hypersensitivity during stress in remitted individuals with recurrent depression. Biol Psychiatry 78: 67-76.

Admon R, Pizzagalli DA (2015). Dysfunctional reward processing in depression. Curr Opin Psychol 4: 114-118.

Beck AT, Steer RA, Ball R, Ranieri W (1996). Comparison of Beck Depression Inventories -IA and -II in psychiatric outpatients. J Pers Assess 67: 588-597.

Blair JR, Spreen O (1989). Predicting premorbid IQ: a revision of the national adult reading test. Clin Neuropsychol 3: 129-136.

Butler AC, Chapman JE, Forman EM, Beck AT (2006). The empirical status of cognitive-behavioral therapy: a review of metaanalyses. Clin Psychol Rev 26: 17-31.

Carl H, Walsh E, Eisenlohr-Moul T, Minkel J, Crowther A, Moore T et al (2016). Sustained anterior cingulate cortex activation during reward processing predicts response to psychotherapy in major depressive disorder. J Affect Disord 203: 204-212.

Chai XJ, Hirshfeld-Becker D, Biederman J, Uchida M, Doehrmann O, Leonard JA et al (2015). Altered intrinsic functional brain architecture in children at familial risk of major depression. Biol Psychiatry.

Choi KS, Riva-Posse P, Gross RE, Mayberg HS (2015). Mapping the 'depression switch' during intraoperative testing of subcallosal cingulate deep brain stimulation. JAMA Neurol 72: 1252-1260.

Cisler JM, Bush K, Steele JS (2014). A comparison of statistical methods for detecting context-modulated functional connectivity in fMRI. Neuroimage 84: 1042-1052.

Collins PY, Patel V, Joestl SS, March D, Insel TR, Daar AS et al (2011). Grand challenges in global mental health. Nature 475: 27-30.

Cranford JA, Shrout PE, Iida M, Rafaeli E, Yip T, Bolger N (2006). A procedure for evaluating sensitivity to within-person change: can mood measures in diary studies detect change reliably? Pers Soc Psychol Bull 32: 917-929.

Crowther A, Smoski MJ, Minkel J, Moore T, Gibbs D, Petty C et al (2015). Resting-state connectivity predictors of response to psychotherapy in major depressive disorder. Neuropsychopharmacology 40: 1659-1673.

Der-Avakian A, Markou A (2012). The neurobiology of anhedonia and other reward-related deficits. Trends Neurosci 35: 68-77.

Dichter GS, Felder JN, Petty C, Bizzell J, Ernst M, Smoski MJ (2009). The effects of psychotherapy on neural responses to rewards in major depression. Biol Psychiatr 66: 886-897.

Dichter GS, Gibbs D, Smoski MJ (2014). A systematic review of relations between resting-state functional-MRI and treatment response in major depressive disorder. J Affect Disord 172C: 8-17. 
Dimidjian S, Barrera M Jr, Martell C, Munoz RF, Lewinsohn PM (2011). The origins and current status of behavioral activation treatments for depression. Annu Rev Clin Psychol 7: 1-38.

Dunlop BW, Binder EB, Cubells JF, Goodman MM, Kelley ME, Kinkead B et al (2012). Predictors of remission in depression to individual and combined treatments (PReDICT): study protocol for a randomized controlled trial. Trials 13: 106.

Ferrari AJ, Charlson FJ, Norman RE, Patten SB, Freedman G, Murray CJ et al (2013). Burden of depressive disorders by country, sex, age and year: findings from the global burden of disease study 2010. PLoS Med 10: e1001547.

First MB, Spitzer RL, Gibbon M, Williams JBW (2002). Structured Clinical Interview for DSM-IV-TR Axis I Disorders, Research Version, Patient Edition. (SCID-I/P). New York State Psychiatric Institute: New York: Biometrics Research.

Forbes EE, Dahl RE (2012). Research Review: altered reward function in adolescent depression: what, when and how? J Child Psychol Psychiatry 53: 3-15.

$\mathrm{Fu}$ CH, Steiner H, Costafreda SG (2013). Predictive neural biomarkers of clinical response in depression: a meta-analysis of functional and structural neuroimaging studies of pharmacological and psychological therapies. Neurobiol Dis 52: 75-83.

Glascher J, Hampton AN, O'Doherty JP (2009). Determining a role for ventromedial prefrontal cortex in encoding action-based value signals during reward-related decision making. Cereb Cortex 19: 483-495.

Haber SN, Knutson B (2010). The reward circuit: linking primate anatomy and human imaging. Neuropsychopharmacology 35: 4-26.

Hamilton MA (1960). A rating scale for depression. J Neurol Neurosurg Psychiatry 23: 56-62.

Hasler G, Drevets WC, Manji HK, Charney DS (2004). Discovering endophenotypes for major depression. Neuropsychopharmacology 29: 1765-1781.

Heller AS, Johnstone T, Light SN, Peterson MJ, Kolden GG, Kalin NH, Davidson RJ (2013). Relationships between changes in sustained fronto-striatal connectivity and positive affect in major depression resulting from antidepressant treatment. $A m \mathrm{~J}$ Psychiatry 170: 197-206.

Heller AS, Johnstone T, Shackman AJ, Light SN, Peterson MJ, Kolden GG et al (2009). Reduced capacity to sustain positive emotion in major depression reflects diminished maintenance of fronto-striatal brain activation. Proc Natl Acad Sci USA 106: 22445-22450.

Hopko DR, Lejuez CW, Ruggiero KJ, Eifert GH (2003). Contemporary behavioral activation treatments for depression: Procedures, principles, and progress. Clin Psychol Rev 23: 699-717.

Jacobson NS, Martell CR, Dimidjian S (2001). Behavioral activation treatment for depression: Returning to contextual roots. Clin Psychol Sci Prac 8: 255-270.

Jacobson NS, Truax P (1991). Clinical significance: a statistical approach to defining meaningful change in psychotherapy research. J Consult Clin Psychol 59: 12-19.

Jenkinson M, Bannister P, Brady M, Smith S (2002). Improved optimization for the robust and accurate linear registration and motion correction of brain images. Neuroimage 17: 825-841.

Jenkinson M, Smith S (2001). A global optimisation method for robust affine registration of brain images. Med Image Anal 5: $143-156$.

Joiner TE, Brown JS, Metalsky GI (2003). A test of the tripartite model's prediction of anhedonia's specificity to depression: patients with major depression versus patients with schizophrenia. Psychiatry Res 119: 243-250.

Kaiser RH, Andrews-Hanna JR, Wager TD, Pizzagalli DA (2015). Large-scale network dysfunction in major depressive disorder: a meta-analysis of resting-state functional connectivity. JAMA Psychiatry 72: 603-611.

Kaiser RH, Whitfield-Gabrieli S, Dillon DG, Goer F, Beltzer M, Minkel J et al (2015). Dynamic resting-state functional connectivity in major depression. Neuropsychopharmacology 41: $1822-1830$.

Kapur S, Phillips AG, Insel TR (2012). Why has it taken so long for biological psychiatry to develop clinical tests and what to do about it? Mol Psychiatry 17: 1174-1179.

Knutson B, Bhanji JP, Cooney RE, Atlas LY, Gotlib IH (2008). Neural responses to monetary incentives in major depression. Biol Psychiatry 63: 686-692.

Knutson B, Fong GW, Adams CM, Varner JL, Hommer D (2001). Dissociation of reward anticipation and outcome with eventrelated fMRI. Neuroreport 12: 3683-3687.

Knutson B, Westdorp A, Kaiser E, Hommer D (2000). FMRI visualization of brain activity during a monetary incentive delay task. Neuroimage 12: 20-27.

Levkovitz Y, Tedeschini E, Papakostas GI (2011). Efficacy of antidepressants for dysthymia: a meta-analysis of placebocontrolled randomized trials. J Clin Psychiatry 72: 509-514.

Liu X, Hairston J, Schrier M, Fan J (2011). Common and distinct networks underlying reward valence and processing stages: a meta-analysis of functional neuroimaging studies. Neurosci Biobehav Rev 35: 1219-1236.

McGrath CL, Kelley ME, Dunlop BW, Holtzheimer PE 3rd, Craighead WE, Mayberg HS (2014). Pretreatment brain states identify likely nonresponse to standard treatments for depression. Biol Psychiatry 76: 527-535.

McGrath CL, Kelley ME, Holtzheimer PE, Dunlop BW, Craighead WE, Franco AR et al (2013). Toward a neuroimaging treatment selection biomarker for major depressive disorder. JAMA Psychiatry 70: 821-829.

McLaren DG, Ries ML, Xu G, Johnson SC (2012). A generalized form of context-dependent psychophysiological interactions (gPPI): a comparison to standard approaches. Neuroimage 61: 1277-1286.

Miller IW, Bishop S, Norman WH, Maddever H (1985). The Modified Hamilton Rating Scale for Depression: reliability and validity. Psychiatry Res 14: 131-142.

Mitterschiffthaler MT, Kumari V, Malhi GS, Brown RG, Giampietro VP, Brammer MJ et al (2003). Neural response to pleasant stimuli in anhedonia: an fMRI study. Neuroreport 14: 177-182.

Morgan JK, Shaw DS, Olino TM, Musselman SC, Kurapati NT, Forbes EE (2016). History of depression and frontostriatal connectivity during reward processing in late adolescent boys. J Clin Child Adolesc Psychol 45: 59-68.

Northoff G (2016). Spatiotemporal psychopathology I: No rest for the brain's resting state activity in depression? Spatiotemporal psychopathology of depressive symptoms. J Affect Disord 190: 854-866.

Phillips ML, Chase HW, Sheline YI, Etkin A, Almeida JR, Deckersbach $\mathrm{T}$ et al (2015). Identifying predictors, moderators, and mediators of antidepressant response in major depressive disorder: neuroimaging approaches. Am J Psychiatry 172: $124-138$

Pizzagalli DA (2011). Frontocingulate dysfunction in depression: toward biomarkers of treatment response. Neuropsychopharmacology 36: 183-206.

Pizzagalli DA, Iosifescu D, Hallett LA, Ratner KG, Fava M (2008). Reduced hedonic capacity in major depressive disorder: evidence from a probabilistic reward task. J Psychiatr Res 43: 76-87.

Pizzagalli DA, Jahn AL, O'Shea JP (2005). Toward an objective characterization of an anhedonic phenotype: a signal-detection approach. Biol Psychiatry 57: 319-327.

Power JD, Barnes KA, Snyder AZ, Schlaggar BL, Petersen SE (2012). Spurious but systematic correlations in functional connectivity MRI networks arise from subject motion. Neuroimage 59: 2142-2154.

Pruessmann KP, Weiger M, Bornert P, Boesiger P (2001). Advances in sensitivity encoding with arbitrary k-space trajectories. Magn Reson Med 46: 638-651. 
Redlich R, Dohm K, Grotegerd D, Opel N, Zwitserlood P, Heindel W et al (2015). Reward processing in unipolar and bipolar depression: a functional MRI study. Neuropsychopharmacology 40: 2623-2631.

Satterthwaite TD, Kable JW, Vandekar L, Katchmar N, Bassett DS, Baldassano CF et al (2015). Common and dissociable dysfunction of the reward system in bipolar and unipolar depression. Neuropsychopharmacology 40: 2258-2268.

Schaefer HS, Putnam KM, Benca RM, Davidson RJ (2006). Eventrelated functional magnetic resonance imaging measures of neural activity to positive social stimuli in pre- and posttreatment depression. Biol Psychiatry 60: 974-986.

Segarra N, Metastasio A, Ziauddeen H, Spencer J, Reinders NR, Dudas $\mathrm{RB}$ et al (2016). Abnormal f'striatal activity during unexpected reward receipt in depression and schizophrenia: relationship to anhedonia. Neuropsychopharmacology 41: 2001-2010.

Shenhav A, Botvinick MM, Cohen JD (2013). The expected value of control: an integrative theory of anterior cingulate cortex function. Neuron 79: 217-240.
Smith SM (2002). Fast robust automated brain extraction. Hum Brain Mapp 17: 143-155.

Smith SM, Jenkinson M, Woolrich MW, Beckmann CF, Behrens TE, Johansen-Berg $\mathrm{H}$ et al (2004). Advances in functional and structural MR image analysis and implementation as FSL. Neuroimage 23 suppl 1: S208-S219.

Smoski MJ, Felder J, Bizzell J, Green SR, Ernst M, Lynch TR et al (2009). fMRI of alterations in reward selection, anticipation, and feedback in major depressive disorder. J Affect Disord 118: 69-78.

Truong TK, Song AW (2008). Single-shot dual-z-shimmed sensitivity-encoded spiral-in/out imaging for functional MRI with reduced susceptibility artifacts. Magn Reson Med 59: 221-227.

Undurraga J, Baldessarini RJ (2012). Randomized, placebo-controlled trials of antidepressants for acute major depression: thirty-year meta-analytic review. Neuropsychopharmacology 37: 851-864.

Zhang WN, Chang SH, Guo LY, Zhang KL, Wang J (2013). The neural correlates of reward-related processing in major depressive disorder: a meta-analysis of functional magnetic resonance imaging studies. J Affect Disord 151: 531-539.

Supplementary Information accompanies the paper on the Neuropsychopharmacology website (http://www.nature.com/npp) 